\title{
Resultados de un programa de ejercicio físico combinado con electroestimulación neuromuscular en pacientes en hemodiálisis
}

\author{
Anna Junque Jiménez, Vicent Esteve Simón, Gorka Iza Pinedo, Esther Tomás Bernabeú, Inés Luceño Soler, \\ Oscar Paz López, Marisa Lavado Sampere, Manel Ramírez de Arellano
}

Servei de Nefrologia Hospital de Terrassa. Consorci Sanitari de Terrassa. Barcelona

\section{Resumen}

Los pacientes en hemodiálisis presentan una importante disminución de la condición física y la capacidad funcional. Ampliamente son conocidos los numerosos beneficios del ejercicio físico en los pacientes con insuficiencia renal en éstos aspectos. Hasta la fecha, existe mínima experiencia con la electroestimulación neuromuscular en los pacientes en hemodiálisis.

\section{Objetivos:}

1. Analizar el efecto de un programa de entrenamiento físico intradiálisis combinado con electroestimulación neuromuscular sobre la fuerza muscular y la capacidad funcional en nuestros pacientes en hemodiálisis.

2. Analizar la seguridad, eficacia y tolerancia de la electroestimulación neuromuscular en nuestros pacientes en hemodiálisis.

\section{Métodos:}

Los pacientes en hemodiálisis realizaron un programa completo de entrenamiento físico mediante balones medicinales, pesas, bandas elásticas y cicloergómetros combinado con electroestimulación neuromuscular en las primeras dos horas de

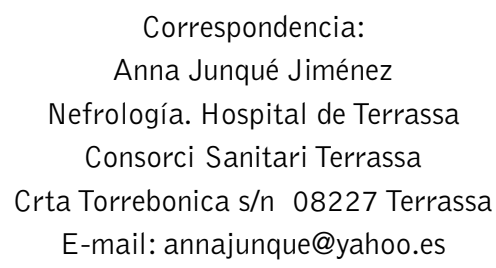

hemodiálisis durante 12 semanas en un estudio prospectivo unicéntrico. La electroestimulación se realizó en ambos cuádriceps usando el Compex® Theta $500 i$.

\section{Principales datos analizados:}

1. Fuerza extensión máxima cuádriceps (FEMQ) y "handgrip" brazo dominante (HG).

2. Test funcionales: "Sit to stand to sit" (STS10) y "six-minutes walking test" (6MWT).

3. Escala Visual Analógica (EVA) y cuestionario propio de electroestimulación neuromuscular (QE).

\section{Resultados:}

11 pacientes incluidos (55\% mujeres). Edad media 67.6 años y 62.3 meses en hemodiálisis. Se observó una mejoría significativa $\left({ }^{*} p<0.05\right)$ en la FEMQ*(13.7 \pm 8.1 vs $16.2 \pm 10.9 \mathrm{~kg}), \mathrm{HG}^{*}(23.8 \pm 15.9$ vs $25.1 \pm 15.9 \mathrm{~kg})$, STS10*(24.6 \pm 13.4 vs $20.1 \pm 10.1$ seg) y $6 \mathrm{MWT}^{*}(21 \%, 332.6$ vs $402.7 \mathrm{~m})$. No observamos dolor muscular, rampas, hormigueos o calambres musculares relevantes en el cuestionario propio de electroestimulación. El grado medio de satisfacción (EVA) fue 9.4 puntos.

\section{Conclusiones:}

1. El programa de entrenamiento físico intradiálisis combinado con electroestimulación neuromuscular mejoró la fuerza muscular y la capacidad funcional en nuestros pacientes en hemodiálisis. 
2. La electroestimulación neuromuscular intradiálisis de ambos cuádriceps resultó segura, efectiva y bien tolerada en nuestros pacientes en hemodiálsis.

3. Éstos resultados constituyen una novedosa alternativa terapéutica relacionada con los beneficios del ejercicio físico en los pacientes en hemodiálisis.

PALABRAS CLAVE:

- HEMODIÁLISIS

- ELECTROESTIMULACIÓN NEUROMUSCULAR

- ENTRENAMIENTO FÍSICO

Exercise training program combined with leg electroestimulation in patients on hemodialysis: a prospective study

\section{Abstract}

Patients on hemodialysis (HD) have a decreased physical and functional capacity. There is proven evidence about the benefits of exercise training on functional capacity in HD. Untildate, minimal experience with electroestimulation (ETEM) in HD patient sis reported.

\section{Objectives:}

1. Analize the effect of intradialysis training program combined with electroestimulation on muscular strenght and functional capacity in our HD patients.

2. Analize the safety, efficacy and tolerance of ETEM in our HD patients.

\section{Methods:}

HD patients were enrolled into an exercise training program combined with ETEM in the first two hours of HD session during a 12 weeks single-center prospective study. ETEM included physical training using balls, weights, elastic bands and cycle ergometer. Leg electroestimulation were performed using the Compex® Theta 500i device in both quadriceps muscles.

\section{Most relevant analized data:}

1. Maximum length quadriceps strength (MLQS) and "hand-grip (HG) dominant arm.

2. Functional capacity tests: "Sit to stand to sit" (STS10) and "six-minutes walking test" (6MWT).

3. Visual analogic scale (VAS) and our own satisfaction electroestimulation questionnaire (SEQ) was completed.

\section{Results:}

11 HD patients included: 55\%women. Mean age 67.6 years and 62.3 months on HD.A significant $\left({ }^{*} p<0.05\right)$ improvement was observed in MLQS* $(13.7 \pm 8.1$ vs $16.2 \pm 10.9 \mathrm{~kg}), \mathrm{HG} *(23.8 \pm 15.9$ vs $25.1 \pm 15.9 \mathrm{~kg}), \mathrm{STS} 10 *(24.6 \pm 13.4$ vs $20.1 \pm 10.1$ $\mathrm{sec})$ and $6 \mathrm{MWT}^{*}(21 \%, 332.6$ vs $402.7 \mathrm{~m})$. No relevant muscular pain, cramps, tingles or pricks were related in the SEQ. The VAS satisfaction degree was 9.4 points.

\section{Conclusions:}

1. Intradialysis training program combined with quadriceps electroestimulation improved muscular strenght and functional capacity in our HD patients.

2. Intradialysis quadriceps electroestimulation was safe, effective and well tolerated in our HD patients.

3. These results represents a wide therapeutic possibility related to the benefits of exercise training for HD patients.

\section{KEYWORDS:}

- HEMODIALYSIS

- NEUROMUSCULAR ELECTROESTIMULATION

- PHYSICAL TRAINING 


\section{Introducción}

Un aspecto de extraordinaria importancia en los pacientes renales es la disminución de la capacidad física conforme avanza la permanencia en hemodiálisis (HD), mediada entre otros factores por el sedentarismo, la elevada morbilidad cardiovascular, la anemia y las alteraciones propias de la uremia en forma de miopatía y neuropatía; conduciendo a la aparición de diversos síntomas musculares que limitan su capacidad física diaria ${ }^{(1,2)}$.

Desde principios de la década de los ochenta, algunos países comenzaron a implantar programas de ejercicio físico durante la HD. Desde entonces hasta hoy, todos los estudios refieren beneficios del ejercicio en este tipo de pacientes tanto a nivel fisiológico, como funcional 0 psicológico ${ }^{(3-6)}$. A pesar de todo, la implantación de programas de ejercicio en unidades de HD no está generalizada.

Recientemente también cobra gran interés el papel inocuo y beneficioso de la electroestimulación neuromuscular (EENM) como tratamiento coadyuvante del ejercicio físico regular aplicado a pacientes en tratamiento renal sustitutivo en HD. Hasta la fecha, escasos estudios acerca del papel de la EENM sobre la fuerza muscular en los pacientes en HD han sido publicados ${ }^{(7-9)}$.

Por tanto, en nuestro estudio pretendemos analizar el efecto de un programa de entrenamiento físico intradiálisis combinado con EENM sobre la fuerza muscular y la capacidad funcional en nuestros pacientes en HD y analizar la seguridad, eficacia y tolerancia de la EENM en nuestros pacientes.

\section{Material y métodos}

El programa de HD periódica de nuestro hospital está formado por un total de 64 pacientes. El organigrama de la unidad incluye 6 grupos principales de pacientes de distribución similar que realizan sus sesiones de HD de más de 3 horas de duración básicamente en turnos alternos (lunes, miércoles, viernes ó martes, jueves, sábado) con un horario previamente establecido (mañana, mediodía y tarde).

Entre los meses de noviembre 2011 a enero 2012, se realizó un estudio unicéntrico prospectivo de 12 semanas de duración aprobado por el Comité Ético de nuestra Institución para observar el efecto de la introducción de un programa de ejercicio físico combinado con
EENM sobre la fuerza muscular y capacidad funcional de nuestros pacientes.

Como criterios de inclusión se establecieron: otorgar el consentimiento informado, tener una edad igual o superior a 18 años, permanencia en HD superior a 3 meses en nuestro centro y estabilidad clínica y hemodinámica en los últimos 3 meses. Los criterios de exclusión establecidos fueron: presencia de evento cardiovascular reciente, imposibilidad física manifiesta para la realización de ejercicio físico, hipotensión habitual sintomática en las sesiones de HD habituales, presencia de acceso vascular interno para HD en extremidades inferiores, ser portador de marcapasos y no otorgar el consentimiento informado por escrito.

Coincidiendo con las visitas médicas trimestrales programadas de nuestros pacientes se analizaron tanto al inicio como al final del estudio, las principales variables demográficas, antropométricas y los principales datos bioquímicos.

Junto a éstas variables, se tomaron medidas del tono muscular de los grupos musculares bíceps humerales y cuádriceps de ambas extremidades en su posición anatómica de referencia mediante centimetría, con una cinta flexible e inextensible y expresada en centímetros sin comprimir los tejidos blandos de la zona.

Del mismo modo se analizaron variables de fuerza muscular, así como variables de capacidad funcional. Para la valoración de la fuerza muscular de las extremidades superiores se utilizó un dinamómetro homologado tipo Jamar (Hand-grip dynamometer) (HG) en el brazo dominante. Se realizó con el sujeto en pie, con los brazos extendidos a lo largo del cuerpo y se le entregó el dinamómetro en ambos brazos indicándole que hiciera la mayor fuerza posible sin apoyar el brazo en el cuerpo. El brazo que presentó una mayor fuerza, fue considerado como brazo dominante. Para la valoración de la fuerza muscular en extremidades inferiores se utilizó un dinamómetro de tracción homologado tipo Kern (Kern CH50 50KG dynamometer). Se estimó la fuerza máxima de extensión de los músculos cuádriceps (FEMQ) de la pierna izquierda. El paciente permanecía sentado en una silla fija de tal forma que la espalda quedaba apoyada en el respaldo y la cadera y la rodilla a $90^{\circ}$. En esta posición se colocaba una cincha de sujeción inextensible a la altura del tercio distal de la tibia y se le pedía al sujeto que hiciera la mayor fuerza posible para realizar la extensión de la extremidad sin agarrarse con los brazos a la silla.Todas las variables de tono y fuerza 
estudiadas fueron realizadas por el mismo profesional a fin de evitar posibles errores en la medición. Los resultados obtenidos representan la media de tres medidas consecutivas.

Las pruebas utilizadas para la valoración de la capacidad funcional fueron el test de los 6 minutos de la marcha (6MWT) y el test STS10 (sit to stand to sit 10). El test 6MWT se realizó con monitorización de las constantes habituales y la saturación de oxigeno mediante pulsioximetría. Consistía en evaluar la máxima distancia recorrida durante un período de 6 minutos a ritmo activo. Transcurrido el tiempo de la prueba se registraba la distancia total recorrida mediante un odómetro homologado. EI Test STS 10 consistía en levantarse y volverse a sentar durante 10 veces consecutivas lo más rápido posible; partiendo de una posición sentada con los brazos pegados al pecho. Se anotaba el tiempo en segundos que se tardaba en realizar el ejercicio.

La intervención consistía en la realización de un programa de ejercicio físico completo intradiálisis, supervisado y dirigido por nuestro personal de enfermería, combinado con electroestimulación muscular en las extremidades inferiores.

El programa de ejercicio físico se realizaba en las dos primeras horas de la sesión de HD, con una duración aproximada de 45-50 minutos y durante dos sesiones semanales. Durante la sesión de HD, tras un breve período de calentamiento se trabajaban de forma específica la capacidad aeróbica, fuerza, coordinación y flexibilidad en diferentes grupos musculares de aquellas extremidades sin acceso vascular funcionante mediante cintas elásticas de resistencia, balones medicinales, pelotas de contracción, tobilleras con peso añadido, mancuernas y pesas lastradas diversas. Los ejercicios eran adaptados a cada paciente según los niveles de intensidad y se ajustaban a la posición que el paciente tenia durante la sesión de HD, intentando realizar el mayor número de repeticiones posibles y variedad de ejercicios en cada sesión de HD, a fin de evitar la monotonía y mantener una motivación constante a lo largo del estudio.

Tras la sesión de ejercicio físico los pacientes realizaban un programa de electroestimulación neuromuscular de los músculos cuádriceps de ambas extremidades inferiores. El dispositivo utilizado era el modelo Compex Rehab Theta $500 \mathrm{i}$, dotado de diversos programas de ejercicio rehabilitador con distintas fases, tipos e intensidad de corriente. Estos dispositivos fueron adaptados a las características de cada paciente lo largo de las 12 semanas. El programa de electroestimulación incluía un programa de tonificación en la primera semana, continuaba con 1 semana de resistencia aeróbica, 2 semanas de rehabilitación amiotrófica, 2 semanas de rehabilitación hipertrófica, 3 semanas de potenciación muscular y finalmente 3 semanas de fuerza resistencia. Cada paciente tenía siempre sus propios electrodos. Éstos se colocaban de forma precisa sobre el punto motor de los vientres musculares del cuádriceps (recto anterior, vaso interno y externo), garantizando la máxima comodidad y eficiencia del programa, logrando la máxima contracción del músculo elegido. La intensidad máxima se conseguía animando al paciente a soportar el nivel de energía de estimulación indolora más elevada posible, consiguiendo una contracción muscular tolerable y efectiva.

Al finalizar el estudio, invitamos a los pacientes a rellenar un cuestionario anónimo, elaborado por nuestro personal médico, para valorar distintos aspectos y los conocimientos adquiridos acerca de la EENM. En una primea parte se valoraba los aspectos técnicos relacionados con la EENM: duración, comodidad, seguridad y dificultad para llevarse a cabo en HD. La segunda parte, valoraba los síntomas relacionados con el EENM: dolor, calambres, hormigueos, escozor o quemor. La valoración era de 1 (menor puntuación / peor nota posible) a 5 (mayor puntuación / mejor nota posible). Para finalizar, se valoró el grado de satisfacción mediante una escala visual analógica (EVA), con puntuaciones del 1 al 10.

Los datos fueron analizados mediante el programa estadístico SPSSv18. Los datos obtenidos se presentan mediante la media y desviación estándar. La comparación entre las variables cuantitativas se realizó mediante el test $T$ student para datos apareados, considerando una significación estadística si $p<0.05$.

\section{Resultados}

Un total de 11 pacientes superaron los criterios de inclusión y fueron analizados. Un paciente fue excluido del estudio, al no asistir a la visita final de control trimestral. El 55\% eran mujeres, tenían una edad media de $67.6 \pm 16.7$ años con un tiempo de permanencia en HD de $62.3 \pm 79.5$ años y un índice de Charlson medio de 8.7 \pm 2.7 . Las principales etiologías de la insuficiencia renal crónica de nuestros pacientes fueron la hipertensión $(33 \%)$, diabetes melitus $(26 \%)$, pielonefritis crónica $(17 \%)$, poliquistosis hepatorenal $(9 \%)$, patología glomerular $(9 \%)$ y enfermedad renal no filiada $(6 \%)$. 


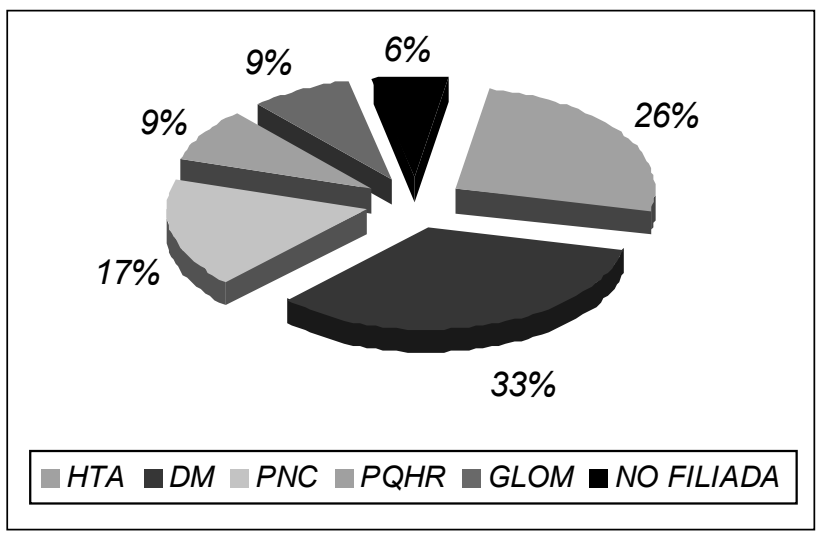

Figura 1. Principales etiologías de la IRCT: HTA: hipertensión, DM: diabetes Melitus, PNC: pielonefritis crónica, PQHR: poliquistosis hepatorenal, GLOM: glomerular y no filiada.

En relación a los principales datos bioquímicos analizados, variables antropométricas y medidas de tono muscular, no se encontraron diferencias significativas tras la realización del ejercicio combinado con EENM (datos no mostrados).

La tabla 1 muestra los resultados relativos a la valoración de la fuerza muscular. Se observó una mejoría significativa de la fuerza muscular tras la realización de ejercicio físico combinado con EENM de las extremidades superiores ( $H G 23.8 \pm 15.9$ vs $25.1 \pm 15.9 \mathrm{~kg}$, $p=0.007$ ) y de las extremidades inferiores (FEMQ $13.7 \pm 8.1$ vs $16.2 \pm 10.9 \mathrm{~kg}, p=0.043$ ).

\begin{tabular}{|c|c|c|c|}
\hline \multirow{2}{*}{} & \multicolumn{2}{|c|}{ Ejercicio } \\
\cline { 2 - 4 } & Inicio & Final & \multirow{2}{*}{} \\
\hline HG $(\mathbf{k g})$ & $23.8 \pm 15.9$ & $25.1 \pm 15.9$ & $0.007^{*}$ \\
\hline FEMQ $(\mathbf{k g})$ & $13.7 \pm 8.1$ & $16.2 \pm 10.9$ & $0.043^{*}$ \\
\hline
\end{tabular}

Tabla 1. Valoración de la fuerza muscular. HG. Hang Grip brazo dominante. FEMQ. Fuerza Extensión máxima del cuádriceps. Significación estadística: * $\mathrm{p}<0.05$.

La tabla 2 muestra los datos relativos a la capacidad funcional. En el test funcional de la marcha (6MWT) observamos un incremento significativo del $21 \%$ en

\begin{tabular}{|c|c|c|c|c|}
\hline & \multicolumn{3}{|c|}{ Ejercicio } & \\
\hline & Inicio & Final & Diferencia & \\
\hline 6mWT(m) & 332.6 & 402.7 & $70.1(21 \%)$ & 0.014 * \\
\hline STS10 (seg) & $24.6 \pm 13.4$ & $20.1 \pm 10.1$ & 4.5 & 0.054 \\
\hline
\end{tabular}

Tabla 2. Valoración de la capacidad funcional. 6MWT : Test de la marcha 6 min. STS10: test sit to stant to sit 10. m: metros; seg: segundos. Significación estadística * $p<0.05$. la distancia recorrida al finalizar el estudio (332.6 \pm 222.1 vs $402.7 \pm 256.5 \mathrm{~m}, p=0.014$ ). En el test funcional del STS10 se observó un menor tiempo en la realización del mismo al finalizar el estudio $(24.6 \pm 13.4$ vs $20.1 \pm 10.1$ seg, $p=0,054$ ), si bien éstas diferencias no alcanzaron la significación estadística preestablecida.

La figura 2 muestra el cuestionario de conocimientos sobre la EENM elaborado por nuestro personal médico. Los resultados relativos a los aspectos técnicos relacionados con el tratamiento neuromuscular se muestran en la figura 3. Ningún paciente encontró dificultad para la realización de la EENM durante las sesiones de HD, 8 pacientes lo consideraron muy seguro, un total de 8 pacientes refirieron una comodidad de la EENM buenamuy buena y la totalidad de los pacientes consideró que la duración del tratamiento era adecuado.

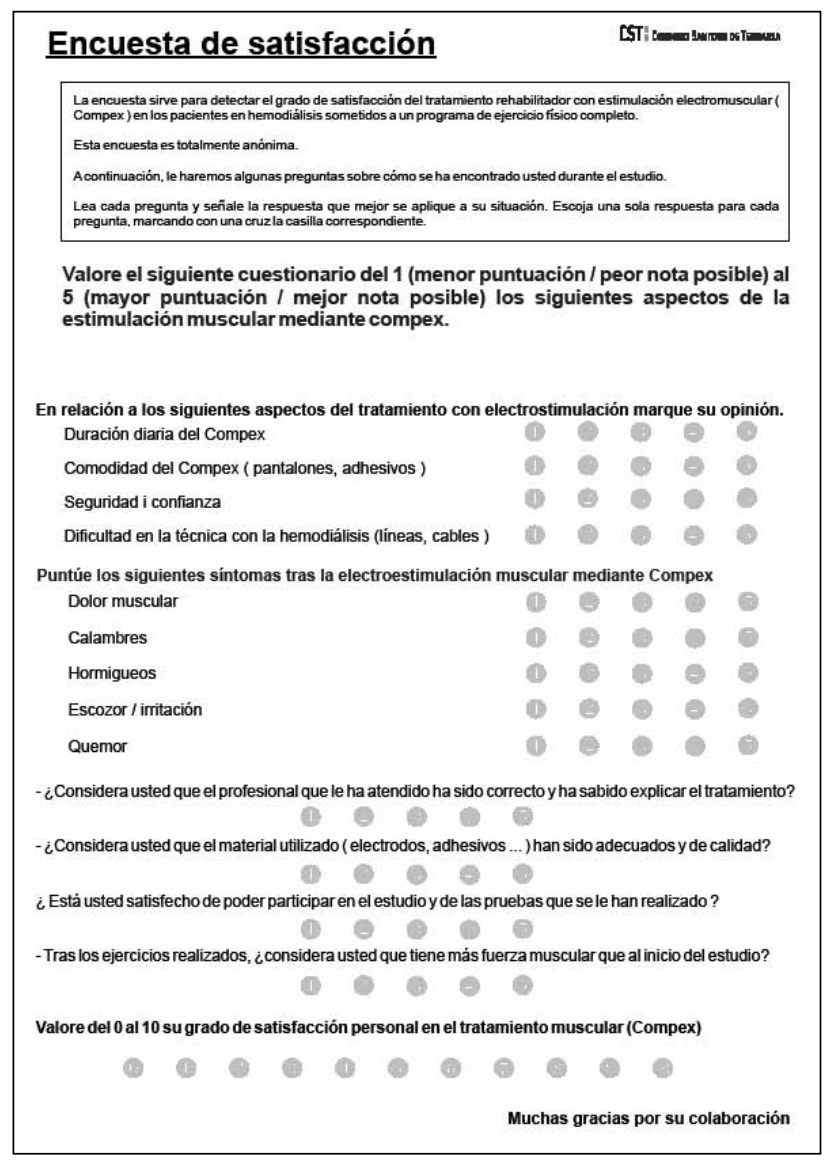

Figura 2. Encuesta de conocimientos y grado de satisfacción sobre electroestimulación neuromuscular (EENM).

De forma global, en cuanto a los síntomas relacionados con el tratamiento neuromuscular (figura 4); muy pocos pacientes mostraron presencia de síntomas asociados con la EENM. Únicamente 1 paciente refirió dolor, 1 


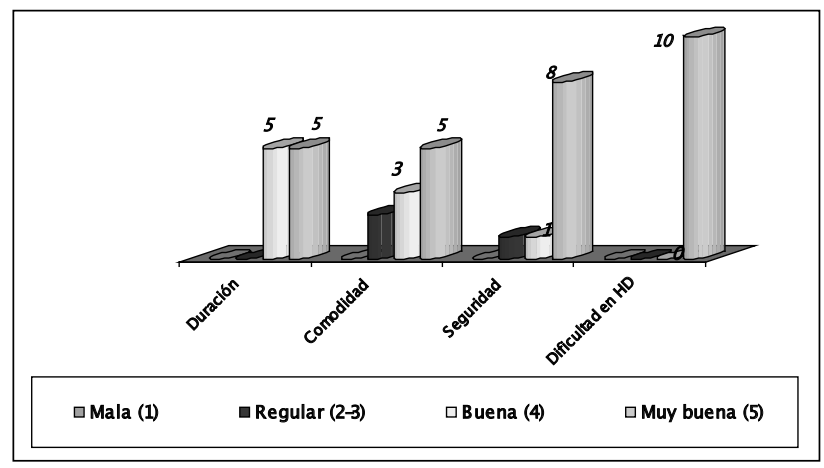

Figura 3. Cuestionario de conocimientos sobre EENM. Aspectos relacionados con el tratamiento neuromuscular: duración, comodidad, seguridad y dificultad en HD.

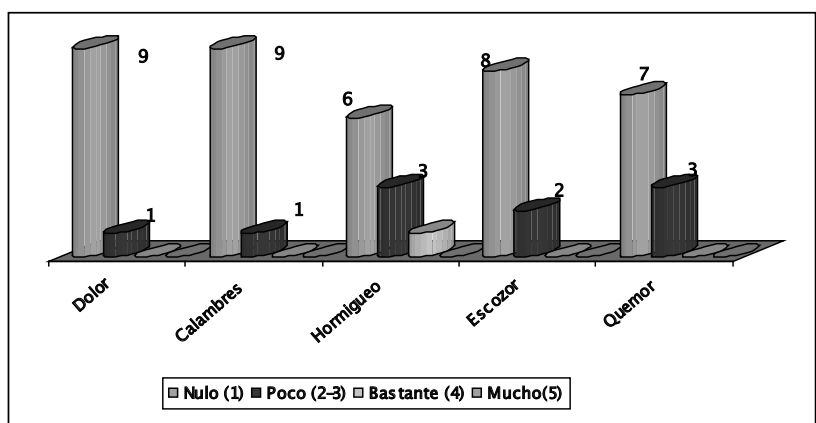

Figura 4.- Cuestionario de conocimientos EENM. Síntomas relacionados con el tratamiento neuromuscular: dolor, calambres, hormigueo, escozor y quemor.

presentó calambres ocasionales, 3 pacientes comentaron tener sensación de hormigueo en las extremidades inferiores, 2 pacientes cierto escozor y 3 pacientes sensación de quemazón. No obstante, todos estos síntomas referidos fueron de baja intensidad y bien tolerados a lo largo del estudio. Finalmente, el grado medio de satisfacción de los pacientes fue de $9.4 \pm 0.5$ puntos en la escala visual analógica.

\section{Discusión}

En las últimas décadas, diversos estudios han sido publicados en relación al ejercicio físico y el paciente renal. Entre las características de todos ellos, destaca la gran heterogeneicidad de los mismos, fundamentalmente en cuanto al tipo y duración del ejercicio realizado así como a los distintos estudiajes del paciente renal; si bien es cierto que la gran mayoría de los estudios se centran en la realización de ejercicio físico de predominio aeróbico durante las sesiones de HD. A pesar de ésta mencionada diversidad, la práctica totalidad de los estudios ponen de manifiesto los efectos beneficiosos del ejercicio físico sobre la fuerza muscular y la capacidad funcional y los aspectos psicológicos ${ }^{(1-6)}$.
En nuestro estudio, la introducción de un programa de ejercicio físico completo combinado con electroestimulación neuromuscular mejoró la fuerza muscular y la capacidad funcional de nuestros pacientes, al igual que los resultados obtenidos en la mayoría de los trabajos publicados en la literatura con anterioridad. Aún con las limitaciones de nuestro estudio, los resultados obtenidos refuerzan los beneficios descritos del ejercicio físico en el paciente en programa de HD, debiendo ser considerado éste como una parte más del cuidado integral del paciente en hemodiálisis a fin de evitar un deterioro progresivo en su condición física y capacidad funcional.

Una menor evidencia científica se encuentra en relación al papel de la electroestimulación neuromuscular en el paciente renal, siendo muy limitados y escasos los estudios publicados en relación a éste tema ${ }^{(7-10)}$. Merece la pena destacar un único estudio publicado a nivel nacional ${ }^{(8)}$ en un grupo de 11 pacientes en programa de HD periódica que mostró una mejoría de la fuerza muscular, la capacidad funcional y la calidad de vida tras la realización de un programa de 5 semanas de duración de ejercicio físico de fuerza - resistencia asociado a electroestimulación neuromuscular de ambos cuádriceps en las sesiones de HD. En este sentido, mencionar que la duración de nuestro estudio fue mayor y que nuestra intervención estaba basada fundamentalmente en la capacidad aeróbica mediante el uso de cicloergómetros además de en la fuerza muscular. A pesar de las diferencias encontradas, con un número de pacientes y población en HD de características similares así como unos test de capacidad funcionales idénticos, los resultados de nuestro estudio son prácticamente superponibles en términos de fuerza muscular y capacidad funcional al de dichos autores, así como al resto de escasos trabajos publicados hasta la fecha actual basados en el uso de cicloergómetros, con períodos de estudio de 12 semanas de duración. Éstos resultados reflejarían por una lado la activación sistémica de grupos musculares de gran tamaño mediante la realización de ejercicio físico y la activación local de las fibras musculares de pequeño tamaño mediante la electroestimulación.

Una de las características diferenciales de nuestro estudio fue analizar la seguridad y tolerancia de la EENM en nuestros pacientes. La electroestimulación neuromuscular se basa en la aplicación de impulsos eléctricos repetitivos de baja frecuencia mediante la colocación de unos electrodos de superficie, consiguiendo la inmediata activación de los diferentes grupos musculares. Ampliamente es utilizada en el ámbito de la de fisioterapia en pacientes en programa rehabilitación muscular. Aunque 
carece de efectos adversos considerables, ésta corriente eléctrica, ocasionalmente puede ser dolorosa ${ }^{(9,10)}$.

De forma global, los pacientes de nuestro estudio refirieron una gran comodidad, seguridad y ausencia de dificultad para realizar la EENM en las sesiones de HD, demostrando un elevado grado de satisfacción. En cuanto a la presencia de síntomas, los más relevantes fueron la sensación de hormigueo y quemazón ligada a la corriente eléctrica aplicada, tal y como era de esperar. El resto de síntomas de nuestro cuestionario fueron percibidos todos ellos en forma de muy baja intensidad y sin ser molestos para los pacientes en ningún caso.

Con los resultados obtenidos, la EENM aplicada en los pacientes renales en $\mathrm{HD}$, podría constituir una alternativa terapéutica eficaz en perfiles seleccionados de pacientes con imposibilidad manifiesta para la realización de ejercicio físico y marcada debilidad muscular; si bien sería necesaria la realización de estudios bien diseñados para establecer el exclusivo papel de la electroestimulación en éste tipo de pacientes.

En conclusión, la introducción de un programa de ejercicio físico completo combinado con electroestimulación neuromuscular mejoró la fuerza muscular y la capacidad funcional de nuestros pacientes. La electroestimulación neuromuscular intradiálisis de ambos cuadriceps resultó segura, efectiva y bien tolerada en nuestros pacientes en HD. En espera de futuros estudios, con diseños adecuados; éstos resultados constituyen una novedosa alternativa terapéutica relacionada con los beneficios del ejercicio físico en los pacientes en HD.

Recibido: 10 Agosto 2013

Revisado: 12 Agosto 2013

Modificado: 18 Agosto 2013

Aceptado: 20 Agosto 2013

\section{Bibliografía}

1. Cheema $B$, Singh $M$. Exercise training in patients receiving maintenance hemodialysis: $A$ systematic review of clinical trials. Am J Nephrol 2005;25(4):352-64.

2. Segura-Ortí E. ejercicio en pacientes en hemodiálisis: revisión sistemática de la literatura. Nefrología 30(2): 236-46, 2010.

3. Cheema B, Abas $H$, Smith $B, 0^{\prime}$ Sullivan A, Chan $M$, Patwardhan $A$, et al. Progressive exercise for anabolism in kidney disease (PEAK): A randomized, controlled trial of resistance training during hemodialysis.J Am Soc Nephrol 2007;18(5):1594601.

4. DePaul V, Moreland J, Eager T, Clase CM. The effectiveness of aerobic and muscle strength training in patients receiving hemodialysis and EPO: A randomized controlled trial. Am J Kidney Dis2002;40(6):1219-29.

5. Goldberg AP, Geltman EM, Hagberg JM, Gavin JR 3rd, Delmez JA, Carney RM, et al. Therapeutic benefits of exercise training for hemodialysis patients. Kidney Int Suppl 1983;16:S303-9.

6. Segura-Ortí E, Rodilla-Alama V, Lisón JF. Physiotherapy during hemodialysis: Results of a progressive resistance-training program. Nefrología 2008;28(1):67-72.

7. Dobsak $P$, Homolka $P$, Svojanovsky et al.Intradialytic electrostimulation of leg extensors may improve exercise tolerance and quality of life in hemodialyzed patients. Artif Organs. 2012 Jan;36(1):71-8.

8. Contreras Martos G, Delgado M, Martínez Villar J, Parra I, Borrego F, Segura P. Eficacia de um programa de entranmiento intradiálisis de fuerza-resistencia em combinación com electroestimulación neueromuscular: mejora de la capacidad funcional, fuerza y calidad de vida Rev Soc Enferm Nefrol 14 (2):112-19. 2011.

9. Heidland A, Fazeli G, Klassen A, Sebekova K, Hennemann $H$, Bahner $U$, Di Iorio B. Neuromuscular electrostimulation techniques: historical aspects and current possibilities in treatment of pain and muscle waisting. Clin Nephrol. 2013 Jan;79Suppl 1:S12-23.

10. Klassen A, Racasan $S$, Gherman-Caprioara M, Kürner B, Blaser C, Bahner U, Heidland A.Hihg Tone external muscle stimulation in end-stage renal disease:effects on quality of life in patients with Peripherals neuropathy. Clin Nephrol. 2013 Jan;79 Suppl 1:S28-33. 